\title{
Editorial
}

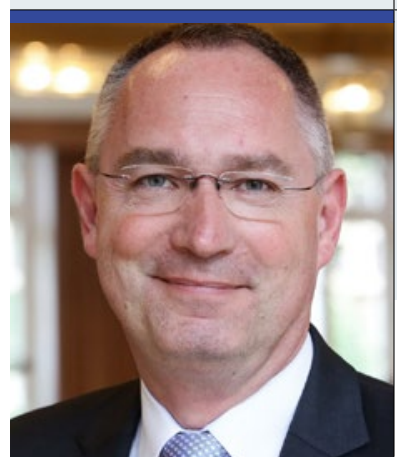

"Die steigende Zahl chronisch schmerzkranker Menschen

ist Beweis dafür, dass die aktuell zugelassenen, verordnungs-

und erstattungsfähigen Arzneimittel allein nicht ausreichen, des

Problems Herr zu werden."

PD Dr. med. Michael A. Überall, Nürnberg

Medizinischer Leiter IFNAP - Institut für Neurowissenschaften,

Algesiologie \& Pädiatrie

\section{Gesetzentwürfe mit erheblicher Sprengkraft}

K ommt der Berg nicht zum Propheten, dann muss der Prophet halt zum Berg kommen. An diese Redensart haben sich sicherlich viele $\mathrm{Ge}$ sundheitsexperten angesichts der erstaunlichen Bewegungsfreudigkeit des Bundesgesundheitsministers bezüglich der Erleichterung des medizinischen Einsatzes von Cannabis und cannabisbasierten Medikamenten erinnert und mit Staunen verfolgt, welches Schauspiel sich da aktuell in Berlin abspielt.

Man könnte fast den Eindruck haben, dass die Gesundheitspolitiker im Deutschen Bundestag verstanden haben, woran es bei der Versorgung chronischer schmerzkranker Menschen in Deutschland unter anderem hakt: an der Widersprüchlichkeit von Verordnungs- und Erstattungsfähigkeit medizinischer Therapien nämlich! Und da nun auch deutsche Gerichte den bislang restriktiven Einsatz von Cannabis bis hin zur Genehmigung des Selbstanbaus erleichtert haben, war es einfach an der Zeit, dass sich endlich auch der Prophet - sprich die Gesundheitspolitik - bewegt.

Nicht anders darf man wohl den aktuell im Gesundheitsausschuss des Deutschen Bundestages diskutierten und in der öffentlichen Anhörung vom 21. September beratenen Entwurf eines „Gesetzes zur Änderung betäubungsmittelrechtlicher und anderer Vorschriften ..." deuten. Vordergründig darauf abzielend, chronisch kranken Menschen den $\mathrm{Zu}-$ gang zu cannabishaltigen Therapien zu erleichtern und gleichzeitig den Selbstanbau zu unterbinden, birgt dieser Gesetzesentwurf darüber hinaus auch das Potenzial, etablierte Strukturen und Prozesse der Verordnungs- und Erstattungsfähigkeit von Arzneimitteln in Deutschland nachhaltig zu verändern. Denn: für Cannabis und cannabisbasierte Arzneimittel liegen aus Sicht des Gemeinsamen Bundesausschuss (G-BA) und des Medizinischen Dienstes der Krankenkassen (MDK) bislang nur für das Fertigarzneimittel Sativex ${ }^{\oplus}$ und die Indikation
„Spastik bei MS“ die für eine arzneimittelrechtliche Zulassung notwendigen kontrollierten Studien vor. Auch an verwertbaren Daten zum (Zusatz-)Nutzen im Vergleich zu anderen bereits etablierten/zugelassenen Therapien mangelt es laut G-BA und MDK. Dass jetzt auch Rezepturarzneien und sogar die Blütentherapie per Gesetz als uneingeschränkt verordnungs- und letztlich auch erstattungsfähig erklärt werden (sollen), zeigt, wie weit die Politik bereit ist auf den Berg medizinischer Versorgungsprobleme chronisch (Schmerz-)Kranker zuzugehen.

Aus Sicht Betroffener ein längst überfälliger Schritt, schließlich ist die kontinuierlich steigende Zahl chronisch schmerzkranker Menschen in Deutschland Beweis dafür genug, dass die aktuell zugelassenen, verordnungs- und als Regelleistung auch erstattungsfähigen Arzneimittel allein nicht ausreichen um des Problems Herr zu werden. Aus Sicht des G-BA und aller analog strukturierten Verbände und insbesondere für die gesetzlichen Krankenkassen ist diese Entwicklung jedoch ein Desaster - denn mit dem „Verstoß“ gegen die etablierten Regeln der Arzneimittelzulassung birgt dieser Gesetzesentwurf beträchtliche Sprengkraft.

Aus Sicht der Schmerzmedizin ist er allemal zu unterstützen, wenngleich es - nicht nur aus arzneimittelrechtlichen Gründen - sicherlich sinnvoll wäre, stärker zwischen den verschiedenen Optionen zu differenzieren und die verfügbare Fertigarznei als Mittel der ersten Wahl in den gesetzesbegleitenden Ausführungsbestimmungen zu positionieren, gefolgt von der THC-Rezepturarznei als zweite und der Blüte als dritte Wahl.

Man darf gespannt sein wie sich der Gesetzesentwurf weiterentwickelt. In jedem Fall kommt hier Bewegung in eine Sache, die lange Zeit für unveränderbar gehalten wurde - und das ist gut so!

PD Dr. med. Michael A. Überall 